\title{
Importance of activated hepatic stellate cells and angiopoietin-1 in the pathogenesis of hepatocellular carcinoma
}

\author{
JI-ZONG LIN $^{1^{*}}$, LI-LI MENG ${ }^{2 *}$, YAN-ZHU LI $^{1}$, SHU-XIAN CHEN $^{1}$, \\ JIAN-LIANG XU ${ }^{1}$, YA-JUN TANG ${ }^{1}$ and NAN LIN ${ }^{1}$ \\ ${ }^{1}$ Department of Hepatobiliary Surgery, Third Affiliated Hospital of Sun Yat-Sen University, Guangzhou, Guangdong 510630; \\ ${ }^{2}$ Department of Gynecology and Obstetrics, Sun Yat-Sen Memorial Hospital of Sun Yat-Sen University, \\ Guangzhou, Guangdong 510000, P.R. China
}

Received June 17, 2015; Accepted May 23, 2016

DOI: $10.3892 / \mathrm{mmr} .2016 .5418$

\begin{abstract}
Previous studies have determined that activated hepatic stellate cells (aHSCs) promote the progression of hepatocellular carcinoma (HCC) by increasing angiogenesis in cancerous tissues. In addition, angiopoietin 1 (Ang-1) has been reported to be involved in tumor growth and metastasis via the promotion of angiogenesis. It remains unclear whether aHSCs and Ang-1 are involved in the angiogenesis in HCC. A total of $25 \mathrm{HCC}$ and tumor-adjacent tissues, and 21 normal liver tissues were used in the present study. Immunohistochemistry (IHC) was used to detect the expression of Ang-1 and $\alpha$ smooth muscle actin ( $\alpha$-SMA). The expression of CD34 was also analyzed using IHC to evaluate the microvessel density (MVD). The protein expression levels of Ang-1 were evaluated using western blot analysis. The association between aHSC, Ang-1 and angiogenesis was determined using Spearman's rank correlation coefficient. The present study determined that the expression of $\alpha$-SMA, Ang-1 and MVD (CD34) was significantly higher in the HCC tissues when compared with tumor-adjacent tissues and normal liver tissues. Spearman's rank analysis identified a positive correlation between the expression of $\alpha$-SMA, Ang-1 and CD34. This suggests that $\alpha$-SMA-positive aHSCs promoted angiogenesis by expressing Ang-1, resulting in the proliferation and metastasis of HCC.
\end{abstract}

\section{Introduction}

Hepatocellular carcinoma (HCC), the dominant form of primary liver cancer, is one of the most prevalent and life-threatening types of cancer worldwide (1-6). Progress has

Correspondence to: Dr Nan Lin, Department of Hepatobiliary Surgery, Third Affiliated Hospital of Sun Yat-Sen University, 600 Tianhe Road, Guangzhou, Guangdong 510630, P.R. China

E-mail: linnan150615@163.com

"Contributed equally

Key words: hepatocellular carcinoma, aHSC, Ang-1, microvessels been made in the investigation of the pathogenesis and treatment of $\mathrm{HCC}$, however, recurrence and metastasis remain key challenges for effective treatment of HCC, thus limiting the prognosis and quality of life of patients with HCC.

Typically, HCC is a hypervascular tumor. Microvessel formation is essential for the growth and metastasis of HCC. Microvessel density (MVD) is positively-associated with the growth and metastasis of HCC (7). Previous studies have reported that activated hepatic stellate cells (aHSCs) promote the growth and metastasis of HCC by accelerating the formation of microvessels in HCC tissues (8-11). However, the mechanism underlying the importance of aHSCs in microvessel formation in HCC remains to be fully elucidated. Angiopoietin-1 (Ang-1) is a growth factor involved in angiogenesis. Previous studies have reported that Ang-1 activates angiogenesis and promotes tumor growth and metastasis by regulating the proliferation and migration of endothelial cells in tumor tissues (12-15).

The association between Ang-1 and aHSC has been previously described. The transcription and production of vascular endothelial growth factor (VEGF) and Ang-1 in aHSCs were increased under hypoxic conditions, and VEGF and Ang-1 promoted aHSC proliferation and extracellular matrix deposition, increasing the migration and chemotaxis of cells (16). Taura et al (17) demonstrated that aHSCs promote angiogenesis in HCC by secreting Ang-1, suggesting that aHSC and Ang-1 are important for the development of HCC (17). However, it remains unclear whether aHSC and Ang-1 are associated with microvessel formation in HCC.

The present study compared the expression of Ang-1, $\alpha$ smooth muscle actin ( $\alpha$-SMA) and MVD (CD34) between $\mathrm{HCC}, \mathrm{HCC}$-adjacent tissues and normal liver tissues to determine the association of Ang-1 and aHSC in the development, in particular in angiogenesis, of HCC.

\section{Materials and methods}

Ethical approval of the study protocol. The current study was approved by the Ethics Committee of Sun Yat-sen University Cancer Center (Guangzhou, China). Signed, informed consent was obtained from each patient prior to the beginning of the current study. 
Patients and tumor tissue samples. A total of 25 patients with HCC, 21 males and 4 females, aged between 29 and 75 years old, were enrolled in the present study. Tissue samples, including $\mathrm{HCC}$ tumor tissues and adjacent non-cancerous tissues $(n=25)$, were obtained from patients that underwent resection of primary HCC at the Third Affiliated Hospital, Sun Yat-sen University (Guangzhou, China) between July and October 2013. The patients had not received preoperative chemotherapy or radiotherapy. Control samples were collected from a total of 21 healthy individuals using the same sample collection protocol. Samples were placed in sterile vials and immediately stored at $-80^{\circ} \mathrm{C}$.

Immunohistochemistry (IHC). Isolated tissues were fixed with $4 \%$ paraformaldehyde for $24 \mathrm{~h}$ and embedded in paraffin. Tissue sections $(5 \mu \mathrm{m})$ were deparaffinized and rehydrated through an ethanol gradient. Antigen retrieval was performed by boiling in a $10 \mathrm{mM}$ sodium citrate buffer ( $\mathrm{pH}$ 6) for $15 \mathrm{~min}$. Non-specific binding was blocked by the addition of $5 \%$ bovine serum albumin (Beyotime Institute of Biotechnology, Haimen, China). The sections were then incubated with monoclonal rabbit anti-Ang-1 (1:200 dilution; Abcam, Cambridge, UK; cat. no. ab8451), monoclonal rabbit anti- $\alpha-S M A$ (rabbit anti- $\alpha$-SMA monoclonal antibody; 1:500 dilution; Gene Tech Co., Ltd., Shanghai, China; cat. no. GM085101) and monoclonal rabbit anti-CD34 (1:100 dilution; Santa Cruz Biotechnology, Inc., Dallas, TX, USA; cat. no. sc-19621) antibodies overnight at $4^{\circ} \mathrm{C}$. Detection was conducted using an horseradish peroxidase (HRP) Detection System (Cell Marque; Sigma-Aldrich, St. Louis, MO, USA) and 3,3'-Diaminobenzidene Substrate kit (Forevergen Biosciences, Guangzhou, China), following the manufacturer's protocol. Subsequent to counterstaining with hematoxylin, the slides were dehydrated, mounted and observed under a light microscope (Olympus Corporation, Tokyo, Japan). Quantitative analysis was performed on the IHC images using Image-Pro Plus software (version 4.5; Media Cybernetics, Inc., Rockville, MD, USA), and the mean optical density (MOD) was determined from five randomly selected areas.

Western blotting. A total of 25 tissue samples were used to confirm the expression profile of Ang-1 by western blot analysis. The samples ( 100 mg each) were homogenized and lysed in $500 \mu \mathrm{l}$ radioimmunoprecipitation assay buffer. Next, the samples were centrifuged at $16,000 \mathrm{x} g$ for $1 \mathrm{~min}$ at $4^{\circ} \mathrm{C}$, the supernatant was collected and was quantified by the Bradford Protein assay kit (Beyotime Institute of Biotechnology). Denatured recombinant Ang-1 protein was electrophoresed by $10 \%$ sodium dodecyl sulfate polyacrylamide gel electrophoresis and transferred to nitrocellulose membranes (Thermo Fisher Scientific, Inc., Waltham, MA, USA). The membranes were then blocked with $5 \%$ non-fat milk in phosphate-buffered saline for $1 \mathrm{~h}$ at room temperature and incubated overnight at $4{ }^{\circ} \mathrm{C}$ with anti-Ang-1 monoclonal antibodies (1:200 dilution) or anti-GAPDH mouse monoclonal antibodies $(1: 1,000$ dilution; Santa Cruz Biotechnology, Inc.; cat. no. sc-25778). The HRP-conjugated goat anti-rabbit $\operatorname{IgG}$ was used as a secondary antibody (1:3,000 dilution; Bio-Rad Laboratories, Inc., Hercules, CA, USA; cat. no. 403005). Immunoreactive bands were detected using an enhanced chemiluminescence kit (Thermo Fisher Scientific, Inc.), according to the manufacturer's protocol. Grayscale detection was quantified using ImageJ software (version 1.43; National Institutes of Health, Bethesda, MD, USA) and normalized to GAPDH.

Statistical analysis. Comparison of differential expression of the proteins in the HCC, tumor-adjacent and normal liver tissues was performed using one-way analysis of variance. Fisher's Least Significant Difference test was utilized for comparisons between groups, and Spearman's correlation coefficient was performed to determine the association between expression of any two proteins of interest. All calculations were performed using SPSS software, version 16.0 (SPSS, Inc., Chicago, IL, USA). $\mathrm{P}<0.05$ was considered to indicate a statistically significant difference.

\section{Results}

Expression levels of $\alpha$-SMA in HCC, tumor-adjacent tissues and normal liver tissues. HSCs in normal liver tissues were not activated. Inflammation and mechanical stimulation may activate HSCs to become aHSCs that express $\alpha$-SMA (10). In the present study, the expression levels of $\alpha$-SMA in HSCs in $\mathrm{HCC}$, tumor-adjacent tissues and normal liver tissues were evaluated using IHC. $\alpha$-SMA-positive cells were detected in the cancer cell nest and hepatic blood sinus (Fig. 1A). The MOD levels of $\alpha$-SMA expression in HCC, tumor-adjacent tissues and normal liver tissues were $(4.56 \pm 0.64) \times 10^{4},(2.71 \pm 0.37) \times 10^{4}$ and $(2.25 \pm 0.48) \times 10^{4}$, respectively. The expression of $\alpha$-SMA in HCC was significantly higher compared with tumor-adjacent $(\mathrm{F}=7.09$; $\mathrm{P}<0.05$; Fig. 1B) and normal liver tissues $(\mathrm{F}=7.42$; $\mathrm{P}<0.05$; Fig. 1B).

Expression levels of Ang-1 in HCC, tumor-adjacent and normal liver tissues. The expression of Ang-1 in HSCs in $\mathrm{HCC}$, tumor-adjacent and normal liver tissues was evaluated using IHC. Ang-1-positive cells were primarily detected in the cancer cell nest and hepatic blood sinus (Fig. 2A). The MOD levels of Ang-1 expression in HCC, tumor-adjacent and normal liver tissues were $(3.11 \pm 0.27) \times 10^{5},(2.28 \pm 0.20) \times 10^{5}$ and $(1.26 \pm 0.15) \times 10^{5}$, respectively. The expression of Ang-1 in $\mathrm{HCC}$ was significantly higher compared with tumor-adjacent $(\mathrm{F}=3.00$; $\mathrm{P}<0.05$; Fig. $2 \mathrm{~B})$ and normal liver tissues $(\mathrm{F}=3.14$; $\mathrm{P}<0.05$; Fig. 2B).

The protein expression levels of Ang-1 in HSCs in HCC, tumor-adjacent and normal liver tissues were analyzed using western blot analysis (Fig. 3A). The MOD levels of Ang-1 expression in $\mathrm{HCC}$, tumor-adjacent and normal liver tissues were $4.33 \pm 1.17,1.62 \pm 0.33$ and $1.60 \pm 0.38$, respectively. The expression of Ang-1 in HCC was significantly higher compared with tumor-adjacent $(\mathrm{F}=2.71 ; \mathrm{P}<0.05 ; \mathrm{Fig} .3 \mathrm{~B})$ and normal liver tissues $(\mathrm{F}=2.74$; $\mathrm{P}<0.05$; Fig. $3 \mathrm{~B})$.

MVD in HCC, tumor-adjacent and normal liver tissues. The MVD (CD34) in HCC, tumor-adjacent and normal liver tissues was determined using IHC analysis of CD34 as previously described (18-21). CD34-positive cells were predominantly detected in the cancer cell nest and hepatic blood sinus (Fig. 4A). The MOD levels of CD34 expression in HCC, tumor-adjacent and normal liver tissues were $(18.3 \pm 0.36) \times 103$, 
Table I. Spearman rank analysis to determine the association between $\alpha$-SMA, Ang-1 and CD34 expression levels in hepatocellular carcinoma tissues, tumor-adjacent tissues and normal liver tissues.

\begin{tabular}{|c|c|c|c|c|c|c|}
\hline & \multicolumn{2}{|c|}{ CD34 } & \multicolumn{2}{|c|}{$\alpha$-SMA } & \multicolumn{2}{|c|}{ Ang-1 } \\
\hline & $\begin{array}{l}\text { Correlation } \\
\text { coefficient }\end{array}$ & P-value & $\begin{array}{l}\text { Correlation } \\
\text { coefficient }\end{array}$ & P-value & $\begin{array}{l}\text { Correlation } \\
\text { coefficient }\end{array}$ & $\mathrm{P}$-value \\
\hline CD34 & 1.000 & - & 0.537 & $<0.001$ & 0.610 & $<0.001$ \\
\hline$\alpha$-SMA & 0.537 & $<0.001$ & 1.000 & - & 0.576 & $<0.001$ \\
\hline Ang-1 & 0.610 & $<0.001$ & 0.576 & $<0.001$ & 1.000 & - \\
\hline
\end{tabular}

$\alpha$-SMA, $\alpha$ smooth muscle actin; Ang-1, angiopoietin 1; correlation coefficient, $\mathrm{r}$.

A

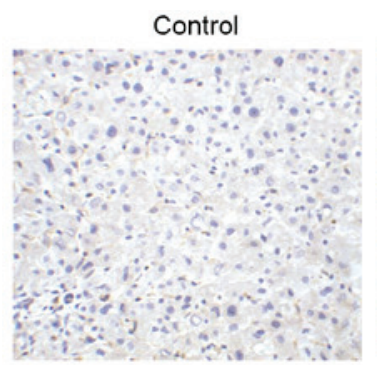

Tumor-adjacent

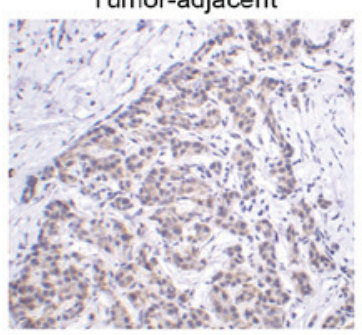

$\mathrm{HCC}$

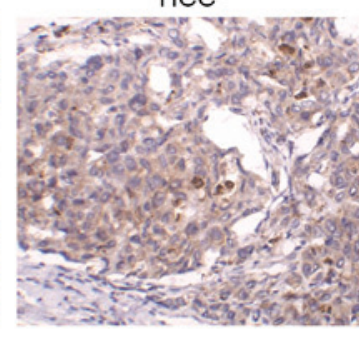

B

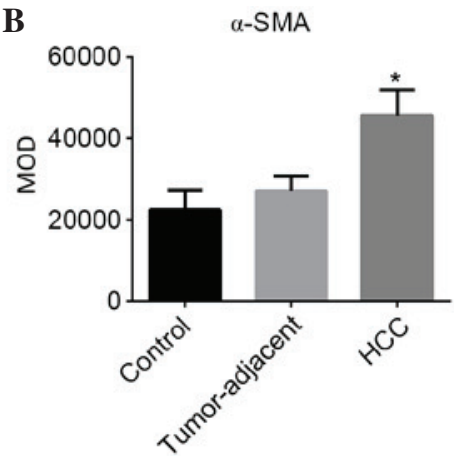

Figure 1. Expression levels of $\alpha$-SMA. (A) Immunohistochemical detection of $\alpha$-SMA expression levels in control, tumor-adjacent and HCC tissues. Magnification, x200. (B) Relative protein expression of $\alpha$-SMA as evaluated by Image-Pro Plus software. *P<0.05 vs. control and tumor-adjacent groups. $\alpha$-SMA, $\alpha$ smooth muscle actin; HCC, hepatocellular carcinoma; MOD, mean optical density.

A

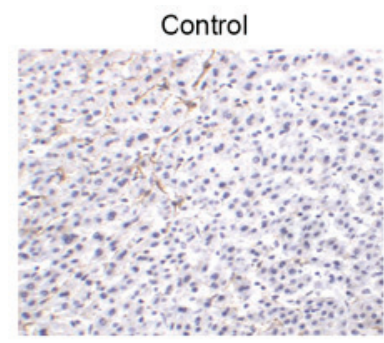

Tumor-adjacent

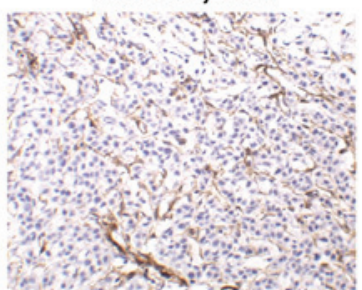

$\mathrm{HCC}$

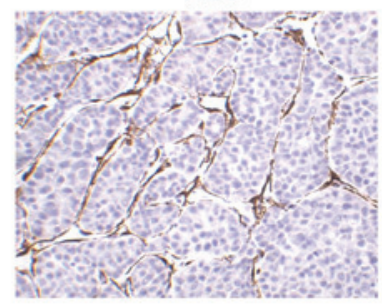

B

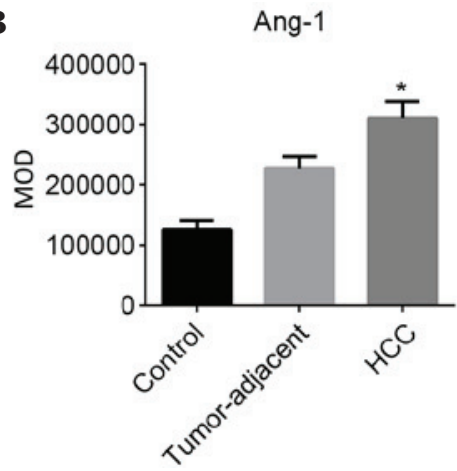

Figure 2. Expression levels of Ang-1. (A) Immunohistochemical detection of Ang-1 expression levels in control, tumor-adjacent and HCC tissues. Magnification, x200. (B) Relative protein expression of Ang-1 as evaluated by Image-Pro Plus software. " $\mathrm{P}<0.05$ vs. control and tumor-adjacent groups. Ang-1, angiopoietin 1; HCC, hepatocellular carcinoma; MOD, mean optical density.

A

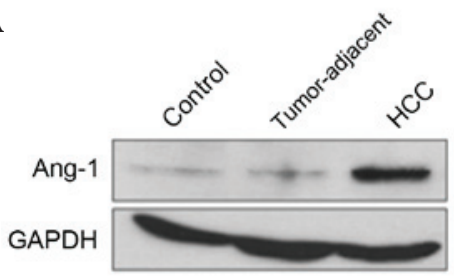

B

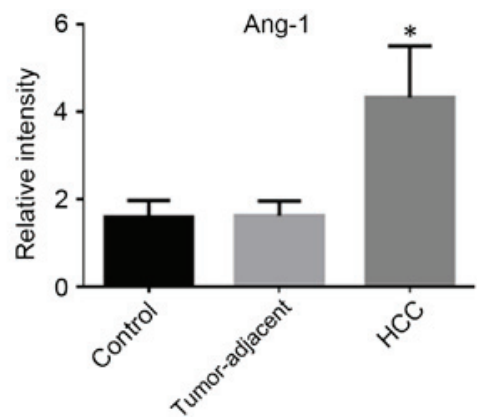

Figure 3. (A) Relative expression of Ang-1 was measured by western blotting, (B) then was quantified ( $\mathrm{n}=25$ ). GAPDH was used as an internal control. * $\mathrm{P}<0.05$ vs. control and tumor-adjacent groups. Ang-1, angiopoietin 1; HCC, hepatocellular carcinoma; GAPDH, glyceraldehyde 3-phosphate dehydrogenase. 
A

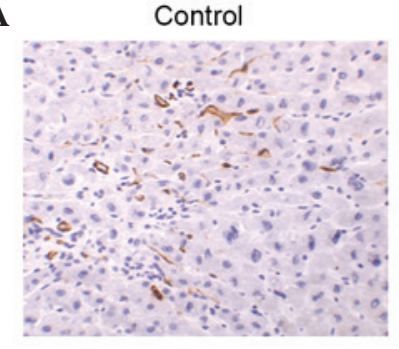

Tumor-adjacent

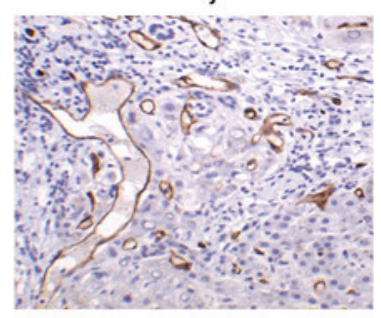

$\mathrm{HCC}$

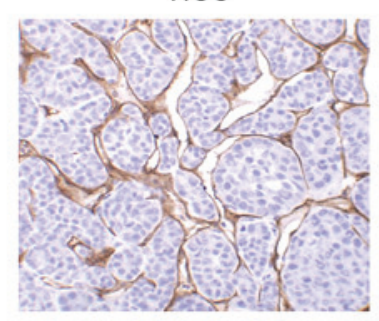

B

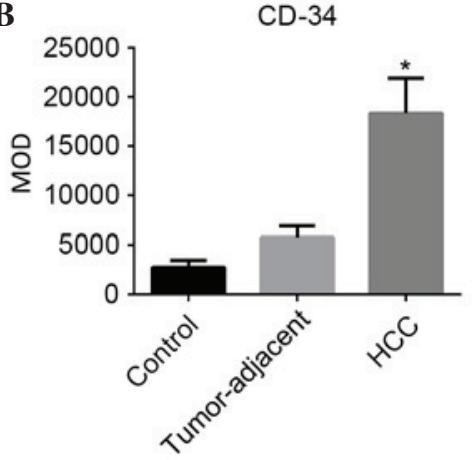

Figure 4. Expression levels of CD34. (A) Immunohistochemical detection of CD34 expression levels in control, tumor-adjacent and gastric cancer tissues. Magnification, x200. (B) Relative protein expression of CD34 as evaluated by Image-Pro Plus software. "P<0.05 vs. control and tumor-adjacent groups. HCC, hepatocellular carcinoma; MOD, mean optical density.

$(5.75 \pm 1.17) \times 103$ and $(2.75 \pm 0.72) \times 103$, respectively. The expression levels of CD34 in HCC were significantly higher compared with tumor-adjacent $(\mathrm{F}=3.21 ; \mathrm{P}<0.05 ; \mathrm{Fig}$. 4B) and normal liver tissues $(\mathrm{F}=3.36$; $\mathrm{P}<0.05$; Fig. 4B).

Associations between the expression levels of Ang-1, $\alpha-S M A$ and MVD (CD34). Spearman's rank correlation coefficient analysis was used to evaluate the association among the expression levels of Ang-1, $\alpha$-SMA and MVD. As presented in Table I, a positive correlation was identified between the expression of Ang-1 and CD34 ( $r=0.610 ; \mathrm{P}<0.05)$, Ang-1 and $\alpha-$ SMA $(r=0.576 ; \mathrm{P}<0.05)$, and $\alpha-S M A$ and CD34 $(r=0.537$; $\mathrm{P}<0.05)$.

\section{Discussion}

HCC is a hypervascular cancer; the development of microvessels is essential for the growth and metastasis of $\operatorname{HCC}(7,22)$. HSCs in normal liver tissues may be activated by inflammation and mechanical stimulation and become activated HSCs $(23,24)$. Activated HSCs proliferate and express $\alpha$-SMA. A previous study reported that aHSCs contributed to the growth and metastasis of HCC by expressing hepatocyte growth factor, interleukin 6 and VEGF (15). However, another previous study has determined that aHSCs additionally inhibit the proliferation and metastasis of HCC cells by expressing laminin 5 and other extracellular matrix components (16). Additionally, aHSCs promote the development of HCC from cirrhosis, HCC growth and metastasis by increasing the formation of microvessels in tumor tissues $(8,9)$. The present study determined that the expression levels of $\alpha$-SMA in aHSCs in HCC tissues were significantly higher compared with tumor-adjacent and normal liver tissues, suggesting that aHSCs may be involved in microvessel formation and development in HCC.

Previous studies have reported that Ang-1 promoted the maturation and maintained the stability of vessels (12-14). In addition, previous studies have determined that Ang-1 regulated the survival, proliferation and metastasis of endothelial cells and promoted the formation of microvessels in tumor tissues $(12,25)$. The expression levels of Ang-1 have been observed to be increased in glioma and ovarian cancers $(26,27)$. The present study determined that the expression of Ang-1 in HCC tissues was significantly higher compared with tumor-adjacent and normal liver tissues. In addition, the MVD in HCC tissues was significantly higher compared with tumor-adjacent and normal liver tissues. These results suggest that Ang-1 is important for microvessel formation in $\mathrm{HCC}$, which is consistent with the results of previous studies (18-21).

Spearman's correlation coefficient analysis identified a positive correlation between the expression levels of $\alpha$-SMA, Ang-1 and CD34, suggesting that Ang-1 and aHSCs are involved in the formation of microvessels in HCC tissues. Ang-1 may also be involved in the regulatory effects of aHSCs on microvessel formation. Previous studies have demonstrated that transcription levels of Ang-1 in aHSCs were increased in response to hypoxia. In addition, Ang-1 may further promote the proliferation and mobility of aHSCs, including the deposition of extracellular matrix (7,16,28-30). Activated HSCs are also important for the development of cirrhosis by expressing Ang-1 and promoting angiogenesis (17). The results of the current study suggest that aHSCs promote the growth and metastasis of HCC by increasing the expression of Ang-1 and angiogenesis.

The present study initially detected the expression of $\alpha$-SMA in HCC tissues using IHC. Positive expression of $\alpha$-SMA suggests the existence of aHSCs in HCC tissues. Next, the expression levels of Ang-1 and CD34 were determined. The results demonstrated that the expression levels of Ang-1 and CD34 in HCC were significantly higher compared with tumor-adjacent and normal liver tissues. The expression of $\alpha$-SMA and CD34 was primarily detected between cancer cell nests; however, the majority of Ang-1 expression was detected in cancer cell nests. In addition, a positive correlation was identified among $\alpha$-SMA, Ang-1 and CD34. In conclusion, the results of the present study suggest that aHSCs increased the expression of Ang-1, resulting in angiogenesis in HCC tissues, thus promoting the growth and metastasis of HCC. However, the molecular mechanisms underlying the role of aHSCs in the development of HCC require further investigation.

\section{Acknowledgements}

The present study was supported by grants from the Basic Research Projects and New Disciplines, Cross 
Disciplinary Funding Program of Sun Yat-Sen University (grant no. 15YKJC19C), the Science and Technology Planning Project of Guangdong Province (grant no. 2016A020212004) and the Natural Science Foundation of Guangdong Province (grant nos. 2014A030313067 and 2014A030313144).

\section{References}

1. European Association For The Study Of The Liver1; European Organisation For Research And Treatment Of Cancer: EASL-EORTC clinical practice guidelines: Management of hepatocellular carcinoma. J Hepatol 56: 908-943, 2012.

2. Cao H, Phan H and Yang LX: Improved chemotherapy for hepatocellular carcinoma. Anticancer Res 32: 1379-1386, 2012.

3. El-Serag HB: Hepatocellular carcinoma. N Engl J Med 365: 1118-1127, 2011.

4. Nordenstedt H, White DL and El-Serag HB: The changing pattern of epidemiology in hepatocellular carcinoma. Dig Liver Dis 42 (Suppl 3): S206-S214, 2010.

5. Roxburgh P and Evans TR: Systemic therapy of hepatocellular carcinoma: Are we making progress? Adv Ther 25: 1089-1104, 2008.

6. El-Serag HB and Rudolph KL: Hepatocellular carcinoma: Epidemiology and molecular carcinogenesis. Gastroenterology 132 2557-2576, 2007.

7. Fernández M, Semela D, Bruix J, Colle I, Pinzani M and Bosch J: Angiogenesis in liver disease. J Hepatol 50: 604-620, 2009.

8. Jia YL, Shi L, Zhou JN, Fu CJ, Chen L, Yuan HF, Wang YF, Yan XL, Xu YC, Zeng Q, et al: Epimorphin promotes human hepatocellular carcinoma invasion and metastasis through activation of focal adhesion kinase/extracellular signal-regulated kinase/matrix metalloproteinase-9 axis. Hepatology 54: 1808-1818, 2011.

9. Sancho-Bru P, Juez E, Moreno M, Khurdayan V, Morales-Ruiz M, Colmenero J, Arroyo V, Brenner DA, Ginés P and Bataller R: Hepatocarcinoma cells stimulate the growth, migration and expression of pro-angiogenic genes in human hepatic stellate cells. Liver Int 30: 31-41, 2010.

10. Amann T, Bataille F, Spruss T, Mühlbauer M, Gäbele E, Schölmerich J, Kiefer P, Bosserhoff AK and Hellerbrand C: Activated hepatic stellate cells promote tumorigenicity of hepatocellular carcinoma. Cancer Sci 100: 646-653, 2009

11. Olaso E, Salado C, Egilegor E, Gutierrez V, Santisteban A, Sancho-Bru P, Friedman SL and Vidal-Vanaclocha F: Proangiogenic role of tumor-activated hepatic stellate cells in experimental melanoma metastasis. Hepatology 37: 674-685, 2003.

12. Holopainen T, Huang H, Chen C, Kim KE, Zhang L, Zhou F, Han W, Li C, Yu J, Wu J, et al: Angiopoietin-1 overexpression modulates vascular endothelium to facilitate tumor cell dissemination and metastasis establishment. Cancer Res 69: 4656-4664, 2009.

13. Ji Y, Wang Z, Li Z, Li K, Le X and Zhang T: Angiotensin II induces angiogenic factors production partly via AT1/JAK2/STAT3/SOCS3 signaling pathway in MHCC97H cells. Cell Physiol Biochem 29: 863-874, 2012.
14. Augustin HG, Koh GY, Thurston G and Alitalo K: Control of vascular morphogenesis and homeostasis through the angiopoietin-Tie system. Nat Rev Mol Cell Biol 10: 165-177, 2009.

15. Huang H, Bhat A, Woodnutt $\mathrm{G}$ and Lappe R: Targeting the ANGPT-TIE2 pathway in malignancy. Nat Rev Cancer 10: 575-585, 2010.

16. Forbes SJ and Parola M: Liver fibrogenic cells. Best Pract Res Clin Gastroenterol 25: 207-217, 2011.

17. Taura K, De Minicis S, Seki E, Hatano E, Iwaisako K, Osterreicher $\mathrm{CH}$, Kodama Y, Miura K, Ikai I, Uemoto S and Brenner DA: Hepatic stellate cells secrete angiopoietin 1 that induces angiogenesis in liver fibrosis. Gastroenterology 135: $1729-1738,2008$

18. Wang XW, Cai CL, Xu JM, Jin H and Xu ZY: Increased expression of chitinase 3-like 1 is a prognosis marker for non-small cell lung cancer correlated with tumor angiogenesis. Tumour Biol 36: 901-907, 2015.

19. Ding S, Li C, Lin S, Yang Y, Liu D, Han Y, Zhang Y, Li L, Zhou L and Kumar S: Comparative evaluation of microvessel density determined by CD34 or CD105 in benign and malignant gastric lesions. Hum Pathol 37: 861-866, 2006.

20. Sluimer JC and Daemen MJ: Novel concepts in atherogenesis: Angiogenesis and hypoxia in atherosclerosis. J Pathol 218: 7-29, 2009.

21. Gong Y, Sun X, Huo L, Wiley EL and Rao MS: Expression of cell adhesion molecules, CD44s and E-cadherin, and microvessel density in invasive micropapillary carcinoma of the breast. Histopathology 46: 24-30, 2005.

22. Risau W: Mechanisms of angiogenesis. Nature 386: 671-674, 1997.

23. Wynn TA: Cellular and molecular mechanisms of fibrosis J Pathol 214: 199-210, 2008.

24. Sokolović A, Sokolović M, Boers W, Elferink RP and Bosma PJ: Insulin-like growth factor binding protein 5 enhances survival of LX2 human hepatic stellate cells. Fibrogenesis Tissue Repair 3: 3, 2010.

25. Stoeltzing O, Ahmad SA, Liu W, McCarty MF, Wey JS, Parikh AA, Fan F, Reinmuth N, Kawaguchi M, Bucana CD and Ellis LM: Angiopoietin-1 inhibits vascular permeability, angiogenesis, and growth of hepatic colon cancer tumors. Cancer Res 63: 3370-3377, 2003.

26. Thomas $\mathrm{M}$ and Augustin HG: The role of the Angiopoietins in vascular morphogenesis. Angiogenesis 12: 125-137, 2009.

27. Machein MR, Knedla A, Knoth R, Wagner S, Neuschl E and Plate KH: Angiopoietin-1 promotes tumor angiogenesis in a rat glioma model. Am J Pathol 165: 1557-1570, 2004.

28. Valfrè di Bonzo L, Novo E, Cannito S, Busletta C, Paternostro C, Povero D and Parola M: Angiogenesis and liver fibrogenesis. Histol Histopathol 24: 1323-1341, 2009.

29. Medina J, Arroyo AG, Sánchez-Madrid F and Moreno-Otero R: Angiogenesis in chronic inflammatory liver disease. Hepatology 39: 1185-1195, 2004.

30. Novo E, Cannito S, Zamara E, Valfrè di Bonzo L, Caligiuri A, Cravanzola C, Compagnone A, Colombatto S, Marra F, Pinzani M and Parola M: Proangiogenic cytokines as hypoxia-dependent factors stimulating migration of human hepatic stellate cells. Am J Pathol 170: 1942-1953, 2007. 